newly independent, but economically hard-pressed states of the former Soviet Union. How much of the reported $\$ 10$ billion deal with Chevron will the Kazakh government devote to cleaning up the environment and improving public health? Will energy-starved Armenia restart the two nuclear reactors shut down in 1989 without making them any more resistant to earthquakes? Is non-Soviet Uzbekistan likely to be any more able than its Communist predecessor to free itself from a chemically ruinous and water-squandering cotton monoculture?

Feshbach and Friendly do not attempt to answer these difficult kinds of questions. Their meticulously researched book does, however, provide an invaluable chronicle of the environmental damage wrought by a system that treated natural resources as inexhaustible free goods and the vast Soviet wilderness as an adversary in the battle to fulfil the next five-year plan.

Anna Scherbakova is at the Center for Russian and Eurasian Studies, Monterey Institute of International Studies, 425 Van Buren Street, California 93940, USA.

\title{
Tales of transformation
}

\section{John Cairns}

Origins of Human Cancer: A Comprehenslve Review. Edited by Joan Brugge, Tom Curran, Ed Harlow and Frank McCormick. Cold Spring Harbor Laboratory Press: 1991. Pp. 204. \$80.

THE Cold Spring Harbor Laboratory has held two meetings on the origins of human cancer, one in 1976 and the other in 1990. A comparison of the two books of proceedings gives a fascinating picture of the development of cancer research over the intervening years.

By 1976, President Nixon's war against cancer was underway, and money was flowing into the search for a cure and into the study of the underlying molecular biology of carcinogenesis. For complicated reasons, there was little enthusiasm for the idea of prevention and so epidemiologists did not share in these riches; but they were the people who knew most about the origins of human cancer, and about half of the 1976 meeting was taken up with their account of the epidemiology of cancer. The rest of the meeting was mostly concerned with the biology of tumour viruses and the mechanisms of chemical carcinogenesis. This was the time that assays such as the Ames test were starting to be used, and it seemed conceivable that they would quickly pinpoint the causes of most forms of cancer, with the search for human tumour viruses identifying the other causes. It is instructive to see how many of these hopes have remained unfulfilled, even though our understanding of the molecular biology of cancer has raced ahead.

First, the disappointments. Since 1976, epidemiologists have had a difficult time; the search for the causes of breast and colon cancer is proving to be unexpectedly difficult, because there seems to be no single factor that could be linked to some plausible mechanism of carcinogenesis and could account for the 452 widely different cancer rates in the West and the Far East. The short-term tests for carcinogenicity have not shown us how to prevent cancer; their predictive value is too low, and it is now not even clear how many of the major cancers in industrialized nations are the result of exposure to environmental mutagens. Even when a cause is known, the knowledge may not be put to use; for example, little has been done to stop tobacco companies extending their business to developing nations, which shows that the world is still not keen on prevention. The reason may be that the war against cancer was instigated by clinicians, and the main thrust has therefore been to develop new forms of treatments. But here too, the objective is proving difficult to reach; readers of Nature have about the same overall chance of dying of cancer next year as their parents had when they were the same age.

Most of the 1990 meeting, however, was concerned with the successes. A few new causes of cancer have been discovered, of which hepatitis $B$ virus and certain strains of human papilloma virus are the most important; together they may account for almost as many cancers as cigarettes. Rid the world of these three agents, and you have solved half the problem.

The transforming oncogenes of DNA and RNA tumour viruses were discussed at the 1976 meeting, but I do not think anyone present guessed how quickly this field was to develop. The turning point came in 1982, with the discovery that human tumours often show changes in genes whose counterparts had been identified in bird and rodent retroviruses. There followed an explosion of information thanks to the evolving technology for identifying and comparing nucleicacid sequences.

About two-thirds of the 1990 meeting was therefore devoted to the function and alterations of oncogenes and tumour-suppressor genes. The functions of the products of these genes are more or less what one might have predicted the transduction of signals between cells and the control of cell division and differentiation - and they operate as signal receptors and transducers or as modulators of enzyme action or as regulators of gene expression. The picture that emerges is both simple and complex. Simple, because the number of such genes is not unmanageably large and certain cancers fairly regularly show alteration in particular oncogenes. Complex, because we now see that the regulation of cell behaviour is achieved by an immensely complicated set of interactions between proteins and nucleic acids, and this adds another dimension to all those already complex diagrams of intermediary metabolism. And there is the further dimension of time; particular patterns of interaction are transient and normally not repeated in the life of an organism, and that is why many of the mutations studied by developmental biologists prove to be in what are now called oncogenes.

We can surely expect that all this bustling molecular biology will soon be illuminating the origins of human cancer. In 1982 , it was already possible to see the day coming when the molecular biologist would be able to look at the sequence changes in each class of cancer and tell the epidemiologist what kind of mutagens to look for in the patient's past. This has now come to pass, although so far the epidemiologists have been told only what they already know - for example, that skin cancers show the kinds of sequence changes in the p53 gene that one would expect to be produced by ultraviolet light, and that familial susceptibility to certain cancers can be associated with germline mutations in tumour-suppressor genes.

The 80 or so articles in the new Origins of Human Cancer give a wonderful overview of these developments. What would I not give for a preview of the discoveries of the next 14 years.

John Cairns, formerly in the Department of Cancer Biology at the Harvard School of Public Health, Boston, USA, is at Holly Grove House, Wilcote, Chipping Norton; Oxfordshire OX7 3EA, UK.

\section{Corrections}

- In his review of The Cybernetics Group (Nature 356, 117; 1992), Donald Michie complained of a lack of references to the writings and activities of "the new cybernetics tradition". The references are in fact to be found in Note 21 on page 326 of the book.

- Andrew Warwick, who reviewed The Maxwellians in Nature 357, 291 (1992), is a fellow at St John's College, University of Cambridge, as well as being affiliated to the Department of History and Philosophy of Science, as reported.

NATURE - VOL 357 - 11 JUNE 1992 